\title{
A Systematic Review and Meta-Analysis of the Efficacy of Piracetam and Piracetam-Like Compounds in Experimental Stroke
}

\author{
Philippa C.R. Wheble Emily S. Sena Malcolm R. Macleod \\ Department of Clinical Neurosciences, University of Edinburgh, Western General Hospital, Edinburgh, UK
}

\section{Key Words}

Piracetam - Oxiracetam • Levetiracetam - GVS-111 •

Noopept $\cdot$ Stroke $\cdot$ Ischaemia $\cdot$ Meta-analysis $\cdot$

Systematic review $\cdot$ Neuroprotection

\begin{abstract}
Background/Objective: Piracetam was a candidate neuroprotective drug for acute stroke ineffective in clinical trial. Here we use systematic review and meta-analysis to describe the evidence supporting a protective effect of piracetam and its derivatives in animal models of stroke. Methods: We present a systematic review of reports describing the use of piracetam and its derivatives in animal models of focal ischaemia, where the outcome was measured as an infarct size or neurological score (Der Simonian and Laird random effects meta-analysis). Results: Only 2 studies, published 10 years after the first clinical trial of piracetam had been initiated, described its efficacy in animal models of stroke. A further 4 studies described the efficacy of related compounds. Piracetam and its derivatives improved the outcome by $30.2 \%(95 \% \mathrm{Cl}=16.1-44.4)$. The median study quality was 4/10 (inter-quartile range $=4-6$ ). Conclusion: Piracetam and its derivatives demonstrate neuroprotective efficacy in experimental stroke, but our findings raise concerns about the amount of available data, the quality of the studies and publication bias.

Copyright $\odot 2007$ S. Karger AG, Basel
\end{abstract}

(c) 2007 S. Karger AG, Basel

$1015-9770 / 08 / 0252-0005 \$ 24.50 / 0$

Fax +41613061234

E-Mail karger@karger.ch

www.karger.com
Accessible online at:

www.karger.com/ced

\section{Introduction}

More than 1,000 drugs have been tested in experimental stroke, with nearly 500 drugs having reported efficacy in animal models of focal cerebral ischaemia [1]. However, there is to date unequivocal evidence for the clinical efficacy of only 2 drugs - asprin [2] and tissue plasminogen activator [3]. This discrepancy has previously been attributed either to falsely positive animal studies or to biological differences between species which restrict the fidelity with which these animal experiments model human disease. However, a recent analysis comparing animal data describing the efficacy of tissue plasminogen activator with clinical trial data suggests that where the design of a clinical trial matches the conditions under which preclinical efficacy was demonstrated, concordance is observed [4]. It may therefore be possible to explain the poor concordance between animal and clinical data on the basis of falsely negative clinical trials [5].

Our ability accurately to interpret the available animal evidence for the efficacy of candidate drugs is crucial to the process of deciding which therapies should be continued into clinical trial and how they should be tested. We therefore set out to establish the range and quality of the animal data supporting the efficacy of piracetam, a candidate neuroprotective drug tested in clinical trial from 1992 in over 1,000 patients.

Systematic review and meta-analysis allow the methodical collection and interpretation of existing data. Systematic review seeks to identify all relevant studies, thereby minimising selection bias. Meta-analysis com-

Dr. Malcolm Macleod

Department of Clinical Neurosciences, University of Edinburgh

Western General Hospital, Crewe Road, Edinburgh EH4 2XU (UK)

Tel. +44 131537 1000, Fax +441313325150

E-Mailmalcolm@apoptosis.freeserve.co.uk 
bines the relevant data from each study, producing a more precise estimate of drug efficacy than is available from individual studies. This is particularly important given the small size - and therefore limited precision - of most animal experiments. The impact of specific study characteristics upon the overall estimate of effect size can then be explored using stratified meta-analysis.

2-Oxo-1-pyrrolidineacetamide (piracetam) has been marketed for many years as a nootropic or 'cognition-enhancing' agent and together with its pyrrolidinone derivatives (levetiracetam, oxiracetam and GVS-111) forms a group of psychoactive substances which have been reported to stimulate neuronal function, enhance cognitive performance [6] and increase neuronal resistance to injury [7]. Piracetam has been reported to increase cerebral blood flow and glucose metabolism in both infarcted and penumbral tissues and to be an effective adjunct to speech therapy in improving language functions in patients with aphasia following stroke [8]. Although there is limited evidence regarding the mechanism(s) through which piracetam may be neuroprotective, it has been argued that this relates to its ability to localize polar heads in the phospholipid bilayer of the cell membrane [9]. This is held to allow for the restoration of membrane fluidity and to facilitate the maintenance of membrane-bound cell functions, including ATP production, neurotransmission and second messenger activity, thereby protecting neurons from ischaemic damage.

These agents have been applied in diverse clinical situations; while piracetam is not widely used in the treatment of epilepsy, it has efficacy against the myoclonus of the progressive myoclonus epilepsies $[10,11]$ and that seen in idiopathic generalized epilepsy [12]. Both oxiracetam [13] and GVS-111 [14] have reported efficacy in the promotion of cognitive function, but this has not been confirmed in large-scale clinical trials. It is not clear whether these other nootropics simply recapitulate the effects of piracetam with greater potency, or whether they have improved or different selectivity in their effects which might suggest different clinical applications. Indeed, there are insufficient clinical trial data to allow any firm conclusions about differences between these nootropic drugs to be drawn.

We set out to explore the strength of evidence for a neuroprotective effect of piracetam in experimental stroke through systematic review and meta-analysis to determine factors which might contribute to the difficulty in translating observed efficacy from bench to bedside so that we might contribute to continuing efforts aimed at further improving preclinical study methodology and the identification of drugs for clinical trial.

\section{Methods}

Systematic review: (1) electronic search of Pubmed (1974 to Feb 2005), Embase (1980 to Feb 2005) and BIOSIS (1969 to Feb 2005) using the search terms: (((piracetam) OR (oxiracetam) OR (levetiracetam) OR (GVS-111) OR (Noopept) OR (2-oxo-1-pyrrolidineacetamide)) AND ((stroke) OR (ischaemia) OR (cerebrovascular) OR (middle cerebral artery) OR (MCA) OR (ACA) OR (anterior cerebralartery) OR(MCAO)) AND animals) NOT (coronary OR myocardia*); (2) hand searching of abstracts of scientific meetings including the Society for Neuroscience and the International Society for Cerebral Blood Flow and Metabolism; (3) references to other studies and unpublished data were requested from the senior authors of our identified resources.

Inclusion criteria: 2 investigators (P.C.R.W., E.S.S.) independently assessed the titles and abstracts identified by these searches, and the findings were collated. Full copies of each of the identified publications were obtained and those describing controlled studies of the effect of piracetam or piracetam-like compounds in animal models of focal cerebral ischaemia where the outcome was measured as infarct size and/or a neurological score were selected for further analysis. Disagreements about the selection of studies were resolved in discussion with a third investigator (M.R.M.).

Methods of review: range and quality of studies - the Stroke Therapy Academic Industry Roundtable (STAIR) recommendations [15] relate to both the range and quality of experimental evidence. We assessed the range of evidence with a modified version of the STAIR criteria and determined if the agent had been tested in: (i) 2 or more laboratories, (ii) 2 or more species, (iii) testing in animals with comorbidities, (iv) male and female animals, (v) permanent and temporary models of ischaemia, (vi) at least $1 \mathrm{~h}$ after vessel occlusion, (vii) at least 2 doses, (viii) a route of drug delivery that would be feasible in man, (ix) both histological and neurobehavioural outcomes measured, (x) outcome measured at least 4 weeks after vessel occlusion (xi) in a larger non-rodent species (e.g. primate).

Further to this we assessed study quality against the CAMARADES (Collaborative Approach to Meta-Analysis of Animal Data from Experimental Stroke) 10-item checklist [16], which comprises (i) publication in a peer-reviewed journal, (ii) statement of control of temperature, (iii) randomization to treatment or control, (iv) blinded induction of ischaemia (i.e. concealment of treatment group allocation at time of induction of ischaemia), (v) blinded assessment of outcome, (vi) avoidance of anaesthetics with marked intrinsic neuroprotective properties, (vii) use of animals with hypertension or diabetes, (viii) sample size calculation, (ix) statement of compliance with regulatory requirements, and (x) statement regarding possible conflicts of interest.

Data extraction: a 'comparison' was defined as 'the assessment of outcome in the treatment and control groups following treatment with a given dose of a drug or vehicle, with treatment commencing at a given time before or after the induction of cerebral ischaemia [16]. Each comparison was performed by extracting data for mean outcome, standard deviation and number of animals per group. Where data were expressed graphically, values were requested from the authors, and where a response was not received, values were carefully read from the graphs. Where the drug was given in multiple doses, the total dose administered in the first $24 \mathrm{~h}$ following induction of ischaemia was recorded. Where neurological tests were performed serially, the final result 
Table 1. Study characteristics

\begin{tabular}{|c|c|c|c|c|c|c|c|c|c|c|}
\hline Author & Year & Drug & Species & $\begin{array}{l}\text { Total } \\
\text { number } \\
\text { of animals }\end{array}$ & $\begin{array}{l}\text { Dose range } \\
\mathrm{mg} / \mathrm{kg}\end{array}$ & $\begin{array}{l}\text { Time of } \\
\text { administra- } \\
\text { tion, min }\end{array}$ & Anaesthetic & $\begin{array}{l}\text { Type of } \\
\text { ischaemia }\end{array}$ & $\begin{array}{l}\text { Route } \\
\text { of } \\
\text { delivery }\end{array}$ & $\begin{array}{l}\text { Outcome } \\
\text { measure(s) }\end{array}$ \\
\hline Kometani et al. [18] & 1991 & oxiracetam & rat & 19 & $100-400$ & 720 & pentobarbital & permanent & IP & infarct volume \\
\hline Ostrovskaya et al. [19] & 1999 & GVS-111 & rat & 14 & 10 & 60 & chloral hydrate & not known & IV & infarct volume \\
\hline Romanova et al. [20] & 2002 & GVS-111 & rat & 48 & 10 & 60 & chloral hydrate & not known & IP & neurological score \\
\hline Tortiglione et al. [21] & 2002 & piracetam & rat & 78 & $375-1,500$ & 360 & chloral hydrate & permanent & IP & infarct volume \\
\hline Xerri et al. [22] & 2003 & piracetam & rat & 20 & 600 & 60 & halothane & permanent & IP & infarct volume \\
\hline
\end{tabular}

Table 2. Reported study quality score

\begin{tabular}{|c|c|c|c|c|c|c|c|c|c|c|c|c|}
\hline Author & Year & 1 & 2 & 3 & 4 & 5 & 6 & 7 & 8 & 9 & 10 & Quality score \\
\hline Hanon and Klitgaard [17] & 2001 & + & + & & & & + & & & + & & 4 \\
\hline Kometani et al. [18] & 1991 & + & & & & & + & + & & + & & 4 \\
\hline Ostrovskaya et al. [19] & 1999 & + & & + & & & + & & & + & & 4 \\
\hline Romanova et al. [20] & 2002 & + & & & & & + & & & & & 2 \\
\hline Tortiglione et al. [21] & 2002 & + & + & + & & + & + & & & + & & 6 \\
\hline Xerri et al. [22] & 2003 & + & + & & & & + & & & + & & 4 \\
\hline
\end{tabular}

was recorded. We also extracted data for the anaesthetic used, time of outcome measurement, method of induction of ischaemia and the individual component items of the quality checklist described above. Two reviewers independently extracted data and differences were resolved by discussion.

Meta-analysis: the data were normalized to outcome in the control group such that complete reversal of the ischaemic injury would give an effect size of $100 \%$. Effect sizes from different experiments were combined using weighted mean difference with a random effects model to give an overall estimate. Where a single control group served multiple treatment groups, the size of the control group was adjusted to account for this. We assessed the effect of a number of variables on effect size by stratifying data according to: drug; drug dosage; time of drug administration; reported quality score; outcome measure; anaesthetic used; route of drug delivery; permanent or temporary ischaemia; use of aged, diabetic or hypertensive experimental animals; method of occlusion; time to outcome measurement; single or multiple dosing regime; and species and gender of animal used. The significance of differences between $\mathrm{n}$ groups was assessed by partitioning heterogeneity and using the $\chi^{2}$ distribution with $n-1$ degrees of freedom (d.f.). To allow for multiple comparisons we set our significance level at $\mathrm{p}<0.001$.

\section{Results}

The initial search identified 467 publications. Of these, 7 (6 full publications and 1 abstract) described the efficacy of nootropic drugs in an animal model of focal cerebral ischaemia and expressed the outcome as a volume of infarction or a neurological score. Hand searching did not identify any further studies, and in response to a request for further relevant information 1 author confirmed that data in an abstract were contained within another included publication. Our analysis is therefore based upon data from 6 full publications describing the efficacy of 4 nootropic drugs (piracetam, levetiracetam, oxiracetam and GVS-111) in experimental stroke, published between 1991 and 2003 [17-22]. Within these 6 studies 16 comparisons were identified describing the outcome in 245 animals (table 1). Five publications reported infarct volume (14 comparisons; 197 animals) and 1 reported a neurobehavioural outcome (2 comparisons; 48 animals). The neurobehavioural data were not analyzed further as the data originate from 1 study.

\section{Study Range and Quality}

The efficacy of levetiracetam, oxiracetam and GVS111 in models of ischaemic stroke was each described in 1 study and 2 studies reported the use of piracetam. There was evidence for efficacy under a range of conditions, meeting 6 of the STAIR criteria. The median reported quality score was 4 (fig. 1; table 2, inter-quartile range $=4-6$ ). No study reported the blinded induction of 


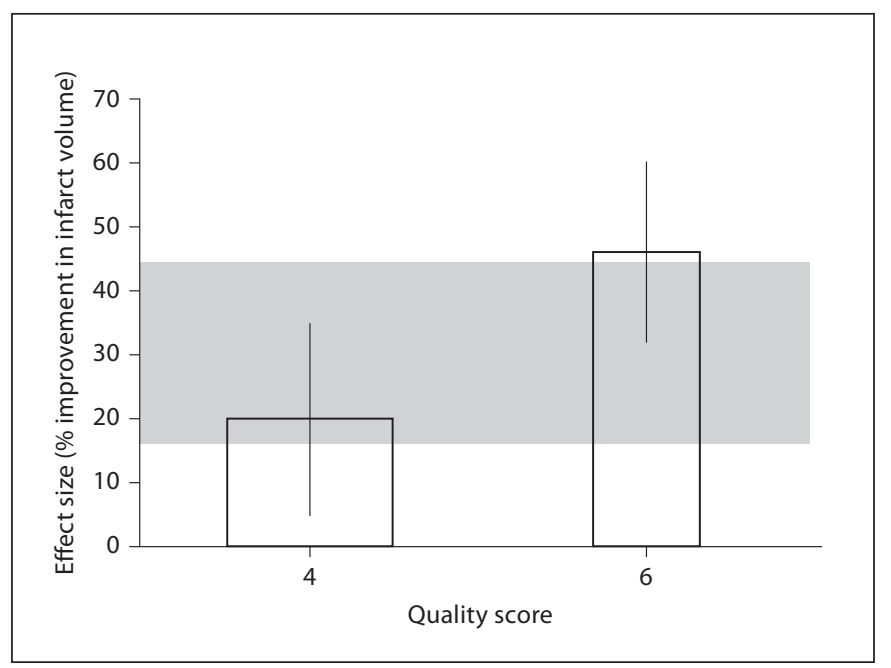

Fig. 1. Effect of study quality on the estimate of efficacy. The shaded grey bar represents the $95 \%$ confidence limits of the global estimate. The vertical error bars represent the $95 \%$ CI for the individual estimates. The width of each vertical bar reflects the log of the number of animals contributing to that comparison. Each stratification accounts for a significant proportion of the heterogeneity observed between studies $(\mathrm{p}<0.001)$.

ischaemia or a sample size calculation and no study included a statement of potential conflict of interest. This is a possible source of bias.

\section{Efficacy}

Overall, nootropic compounds were reported to reduce the infarct volume by $30.2 \%$ (95\% CI $=16.1-44.4$ : $\chi^{2}=47$, d.f. $=13, p<10^{-5}$; fig. $\left.2 \mathrm{a}\right)$. There was good evidence for the efficacy of piracetam (48.5\% reduction in infarct volume; $95 \% \mathrm{CI}=34.9-62.1)$, but for GVS-111 and levetiracetam the 95\% CI were wide and no conclusions can be drawn (GVS-111: 51.1\%; 95\% CI $=11.8$ 90.5; levetiracetam: $20.3 \%$; $95 \% \mathrm{CI}=7.6-33.0)$, and oxiracetam was not effective $(-1.8 \%$; $95 \% \mathrm{CI}=-15.5$ to 11.8 ; fig. 2b). The efficacy appeared to be significantly higher in healthy compared with hypertensive animals $\left(\chi^{2}=\right.$ 27 , d.f. $=13, \mathrm{p}<0.001$; fig $3 \mathrm{a}$ ), when the assessment of outcome was blinded $\left(\chi^{2}=25\right.$, d.f. $=12, p<0.001$; fig. $\left.3 \mathrm{~b}\right)$ and when allocation to group was random $\left(\chi^{2}=29\right.$, d.f. $=13, \mathrm{p}<0.001$; fig. $3 \mathrm{c}$ ). The reported efficacy was highest when halothane anaesthesia was used $\left(\chi^{2}=42\right.$, d.f. $=$ $13, p<0.001$; fig $3 \mathrm{~d}$ ) and when the outcome was assessed between 2 and 7 days $\left(\chi^{2}=30\right.$, d.f. $=13, p<0.001$; fig. 3e).
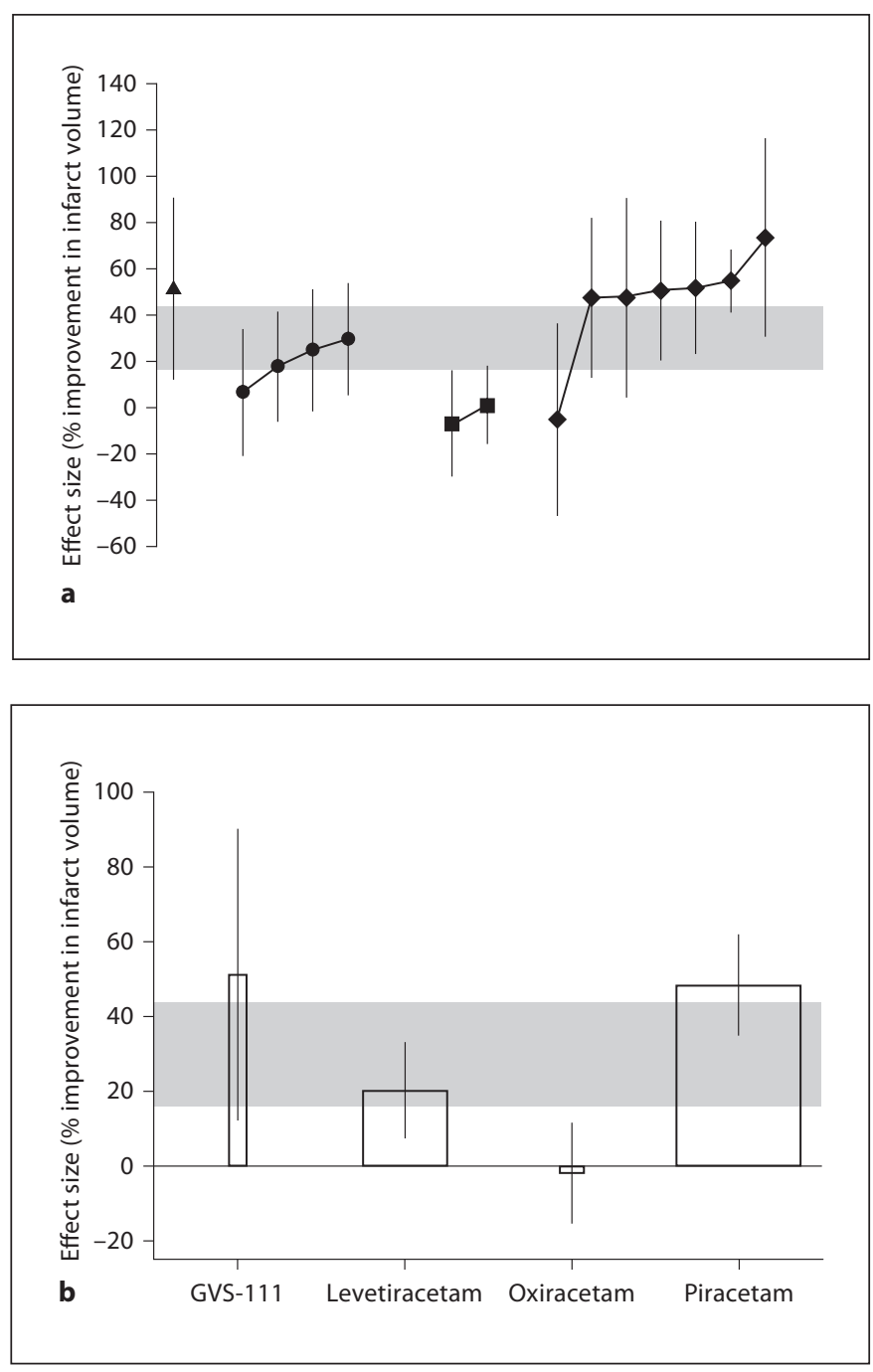

Fig. 2. a Individual comparisons ranked according to their effect on infarct volume. The shaded grey bar represents the $95 \%$ confidence limits of the global estimate. The vertical error bars represent the 95\% CI for the individual estimates. $\boldsymbol{\Delta}=$ GVS-111; $=$ levetiracetam; $\mathbf{\square}=$ oxiracetam; $\boldsymbol{\nabla}$ = piracetam. b Effect of drug used on the estimate of efficacy. The shaded grey bar represents the $95 \%$ confidence limits of the global estimate. The vertical error bars represent the $95 \%$ CI for the individual estimates. The width of each vertical bar reflects the log of the number of animals contributing to that comparison. There was significant betweenstudy heterogeneity for the analysis of drug used $\left(\chi^{2}=40\right.$, d.f. $=$ $13, \mathrm{p}<0.001)$

There was no significant effect of the route of drug administration, the type of ischaemia, the method of induction of middle cerebral artery occlusion or the time of drug administration (not shown), and there were no data from animals other than male rats. 

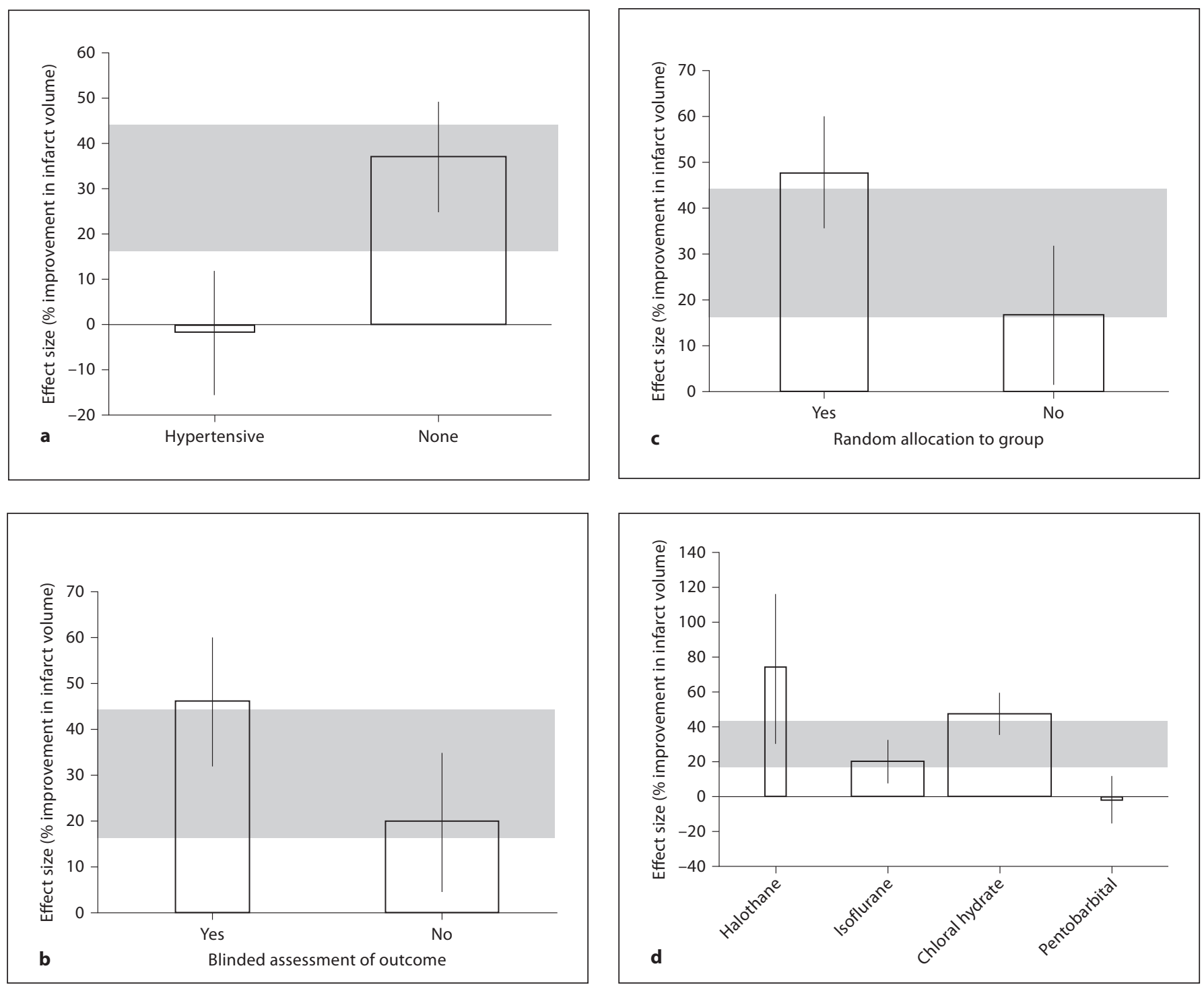

Fig. 3. Effect of comorbidity (a), blinded assessment of outcome (b), random allocation to group (c), anaesthetic used (d) and delay to outcome assessment on the estimate of efficacy (e). The shaded grey bar represents the $95 \%$ confidence limits of the global estimate. The vertical error bars represent the $95 \%$ CI for the individual estimates. The width of each vertical bar reflects the log of the number of animals contributing to that comparison. Each stratification accounts for a significant proportion of the heterogeneity observed between studies $(\mathrm{p}<0.001)$.

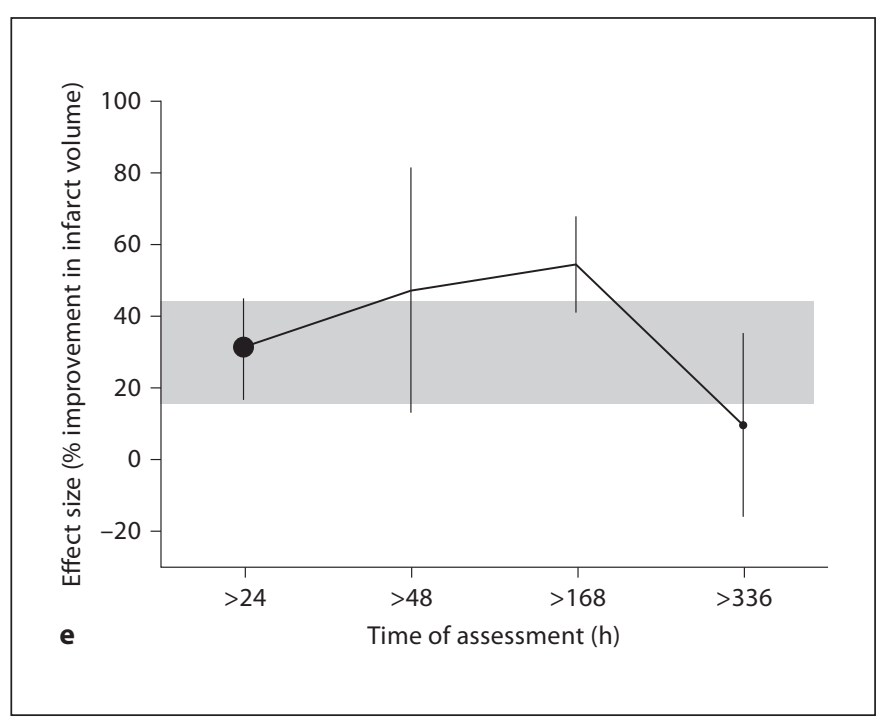




\section{Discussion}

Effects of Piracetam in Animal Models of Stroke

Piracetam and related compounds appear to improve the outcome in animal models of stroke. However, of the 6 studies included in this analysis, only 2 of these related to piracetam [21], and it is difficult to draw robust conclusions from such limited data. The first article was published 10 years after the initiation of the major clinical trial of piracetam in acute stroke [23], suggesting that the decision to progress to clinical trials for piracetam was not based on animal data. It is possible that unpublished data may have been available to those mounting the clinical trial, but it seems unlikely that these data would have remained unpublished for 10 years. A similar observation has been made regarding clinical trials of nimodipine in acute stroke [24]. The failure of clinical trials of both piracetam and nimodipine cannot therefore be taken as a failure of animal modelling of stroke, given that the decision to proceed to clinical trial cannot have been based on published data from animal experiments. Indeed, it may be that had the in vivo efficacy of these compounds been adequately explored (and even now there are insufficient data to allow this), the clinical trials might not have been carried out or they might have been designed differently. For instance, while there are no animal data describing the efficacy of piracetam given more than $6 \mathrm{~h}$ after stroke onset, the largest clinical trial allowed a delay to treatment of $12 \mathrm{~h}$.

\section{Limitations of This Analysis}

There are limitations to our approach. For many candidate neuroprotective drugs there is evidence for a substantial publication bias such that neutral studies appear to be less likely to be published than those reporting positive findings $[25,26]$. With regard to nootropics, there are too few studies reliably to exclude even a major publication bias. If such a bias exists, as seems probable, we may substantially have overstated efficacy. Further, our search strategy may not have identified all relevant publications, particularly as some of these might date from prior to the introduction of electronic biomedical database indexing in the late 1960s.

Biological and systematic sources of bias in individual studies are reflected within the global estimate of outcome, perhaps causing an overstatement of effect size. In contrast to other reviews of neuroprotective drugs [27], higher-quality studies gave higher estimates of efficacy. However, there was an association between study quality and drug (with studies involving piracetam, rather than other nootropics, being of higher quality) and it is plausible that the increased efficacy is due to the drug used and not to study quality. The importance of individual quality scale items in the reduction of biological and systematic bias is therefore not clear and we are exploring this further, in multivariate analysis, across a range of candidate neuroprotective drugs.

While we prespecified the sub-group analysis to be performed and adopted a conservative significance level, some of our findings may have arisen by chance, or to bias or confounding factors as described above, and so these data should be interpreted with caution.

\section{Translation to Clinical Trials}

Between 1988 and 1996, over 5,000 stroke patients were randomized in clinical trials of piracetam [23], tirilazad [28] and clomethiazole [29]; each of these studies produced at best a neutral outcome. A Cochrane review of piracetam [30] reported a non-significant 31\% $(95 \% \mathrm{CI}=-5$ to $+81 \%)$ increase in mortality at 1 month. A post-hoc analysis of data from the Piracetam Acute Stroke Study suggested that piracetam might have a beneficial effect if treatment was initiated within $7 \mathrm{~h}$ of stroke onset [23], where indeed there are at least limited animal data supporting efficacy. The importance of concordance in the time to treatment in translating efficacy from animal studies to clinical trial echoes our findings for tirilazad [4]; animal data suggested a substantial efficacy not seen in clinical trial, but the interval between stroke onset and treatment initiation was substantially longer in clinical (median around $5 \mathrm{~h}$ ) than in animal (median $10 \mathrm{~min}$ ) studies. The hypothesis that piracetam given soon after stroke onset would be effective is consistent with the available animal data and was tested in the Piracetam in Acute Stroke Study II; unfortunately the results of this study have not been published.

\section{Conclusion}

While the overall estimate of efficacy from this metaanalysis suggests that piracetam and piracetam-like compounds have substantial neuroprotective action in models of focal cerebral ischaemia, the data for piracetam are based on only 98 animals, were published at least 10 years after clinical trials had been initiated, and those clinical trials tested efficacy at delays to treatment for which there 
were no supporting animal data. The introduction of candidate neuroprotective drugs to clinical trial should be based on a much more rigorous analysis of animal data if these are to be used to justify proceeding to clinical trial.

\section{Acknowledgements}

We are grateful to Dr. Ioulia Matskevich for translating the Russian publications and for her assistance in contacting the Russian authors.

\section{References}

$\checkmark 1$ O'Collins VE, Macleod MR, Donnan GA, Horky LL, van der Worp BH, Howells DW: 1026 experimental treatments in acute stroke. Ann Neurol 2006;59:467-477.

$\checkmark 2$ Chen ZM, Sandercock P, Pan HC, Counsell C, Collins R, Liu LS, Xie JX, Warlow C, Peto $\mathrm{R}$; CAST and IST collaborative groups: Indications for early aspirin use in acute ischemic stroke: a combined analysis of 40,000 randomized patients from the Chinese acute stroke trial and the international stroke trial. Stroke 2000;31:1240-1249.

-3 National Institute of Neurological Disorders and Stroke rt-PA Stroke Study Group: Tissue plasminogen activator for acute ischemic stroke. N Engl J Med 1995;333:1581-1587.

4 Sena E, Wheble P, Sandercock P, Macleod M: Systematic review and meta-analysis of the efficacy of tirilazad in experimental stroke. Stroke 2007;38:388-394.

$>5$ Jonas S, Aiyagari V, Vieira D, Figueroa M: The failure of neuronal protective agents versus the success of thrombolysis in the treatment of ischemic stroke: the predictive value of animal models. Ann NY Acad Sci 2001;939:257-267.

6 Giurgea C: Pharmacology of integrative activity of the brain: attempt at nootropic concept in psychopharmacology (in French). Actual Pharmacol (Paris) 1972;25:115-156.

7 Gouliaev AH, Senning A: Piracetam and other structurally related nootropics. Brain Res Brain Res Rev 1994;19:180-222.

$\checkmark 8$ Kessler J, Thiel A, Karbe H, Heiss WD: Piracetam improves activated blood flow and facilitates rehabilitation of poststroke aphasic patients. Stroke 2000;31:2112-2116.

9 Peuvot J, Schanck A, Deleers M, Brasseur R: Piracetam-induced changes to membrane physical properties: a combined approach by 31P nuclear magnetic resonance and conformational analysis. Biochem Pharmacol 1995; 50:1129-1134.

-10 Fedi M, Reutens D, Dubeau F, Andermann E, D'Agostino D, Andermann F: Long-term efficacy and safety of piracetam in the treatment of progressive myoclonus epilepsy. Arch Neurol 2001;58:781-786.
1 Koskiniemi M, Van Vleymen B, Hakamies L, Lamusuo S, Taalas J: Piracetam relieves symptoms in progressive myoclonus epilepsy: a multicentre, randomised, double blind, crossover study comparing the efficacy and safety of three dosages of oral piracetam with placebo. J Neurol Neurosurg Psychiatry 1998;64:344-348.

12 Khani YA, Andermann F, Andermann E: Antimyoclonic efficacy of piracetam in idiopathic generalized epilepsy. Epilepsia 2005; 46:1145-1146

13 Aldenkamp AP, van Wieringen A, Alpherts WC, van Emde BW, Haverkort HA, de Vries J, Meinardi H: Double-blind placebo-controlled, neuropsychological and neurophysiological investigations with oxiracetam (CGP 21690E) in memory-impaired patients with epilepsy. Neuropsychobiology 1990;24: 90-101.

14 Ostrovskaya R: Prolyl-containing dipeptide noopept-potential therapeutics of Alzheimer disease. Acta Pharmacol Sin 2006;27 (suppl 1):372.

15 Fisher M: Recommendations for advancing development of acute stroke therapies: Stroke Therapy Academic Industry Roundtable 3. Stroke 2003;34:1539-1546.

16 Macleod MR, O'Collins T, Howells DW, Donnan GA: Pooling of animal experimental data reveals influence of study design and publication bias. Stroke 2004;35:1203-1208.

17 Hanon E, Klitgaard H: Neuroprotective properties of the novel antiepileptic drug levetiracetam in the rat middle cerebral artery occlusion model of focal cerebral ischemia. Seizure 2001;10:287-293.

18 Kometani M, Okada M, Takemori E, Hasegawa Y, Nakao N, Inukai T: Effect of oxiracetam on cerebrovascular impairment in rats. Arzneimittelforschung 1991;41:684-689.

19 Ostrovskaya RU, Romanova GA, Barskov IV, Shanina EV, Gudasheva TA, Victorov IV, Voronina TA, Seredenin SB: Memory restoring and neuroprotective effects of the proline-containing dipeptide, GVS-111, in a photochemical stroke model. Behav Pharmacol 1999;10:549-553.

-20 Romanova GA, Gudasheva TA, Ostrovskaya RU, Shakova FM: Effects of noopept in the photochemically induced stroke model. Biopolymers 2003;71:382.
21 Tortiglione A, Minale M, Pignataro G, Amoroso S, DiRenzo G, Annunziato L: The 2oxopyrrolidinacetamide piracetam reduces infarct brain volume induced by permanent middle cerebral artery occlusion in male rats. Neuropharmacology 2002;43:427-433.

22 XerriC, Zennou-Azougui Y, CoqJO: Neuroprotective effects on somatotopic maps resulting from piracetam treatment and environmental enrichment after focal cortical injury. ILAR J 2003;44:110-124.

-23 De Deyn PP, Reuck JD, Deberdt W, Vlietinck R, Orgogozo JM; Piracetam in Acute Stroke Study (PASS) Group: Treatment of acute ischemic stroke with piracetam. Stroke 1997; 28:2347-2352.

24 Horn J, de Haan RJ, Vermeulen M, Luiten PG, Limburg $M$ : Nimodipine in animal model experiments of focal cerebral ischemia: a systematic review. Stroke 2001;32: 2433-2438.

25 Van der Worp HB, Sena ES, Donnan GA, Howells DW, Macleod MR: Hypothermia in animal models of acute ischaemic stroke: a systematic review and meta-analysis. Brain 2007; in press.

26 Perel P, Roberts I, Sena E, Wheble P, Briscoe C, Sandercock P, Macleod M, Mignini LE, Jayaram P, Khan KS: Comparison of treatment effects between animal experiments and clinical trials: systematic review. BMJ 2007;334:197.

27 Macleod MR, O'Collins T, Horky LL, Howells DW, Donnan GA: Systematic review and metaanalysis of the efficacy of FK506 in experimental stroke. J Cereb Blood Flow Metab 2005;25:713-721.

28 Tirilazad International Steering Committee: Tirilazad mesylate in acute ischemic stroke: a systematic review. Stroke 2000;31:22572265.

29 Lyden P, Shuaib A, Ng K, Levin K, Atkinson RP, Rajput A, Wechsler L, Ashwood T, Claesson L, Odergren T, Salazar-Grueso E: Clomethiazole Acute Stroke Study in ischemic stroke (CLASS-I): final results. Stroke 2002; 33:122-128.

30 Ricci S, Celani MG, Cantisani AT, Righetti E: Piracetam for acute ischaemic stroke. Cochrane Database Syst Rev 2006;19: CD000419. 